\title{
Elucidation of the molecular interactions that enable stable interaction between HIV protease inhibitor ritonavir and human DNA repair enzyme ALKBH2: a molecular dynamics simulation study
}

\author{
Monisha Mohan, Roy Anindya* \\ Department of Biotechnology, Indian Institute of Technology Hyderabad, Kandi - 502284, \\ Sangareddy, Telangana, INDIA \\ * corresponding author: Roy Anindya, E-mail: anindya@bt.iith.ac.in
}




\begin{abstract}
The human DNA repair enzyme AlkB homologue-2 and 3 (ALKBH2 and ALKKBH3) repairs methyl adducts from genomic DNA. Overexpression of ALKBH2 and ALKBH3 has been implicated in both tumorigenesis and chemotherapy resistance in some cancers, including glioblastoma and renal cancer rendering it a potential therapeutic target and a diagnostic marker. However, no inhibitor is available against these important DNA repair proteins. Intending to repurpose a drug as an inhibitor of $A L K B H 2 / A L K B H 3$, we performed in silico evaluation of HIV protease inhibitors and identified Ritonavir as an ALKBH2-interacting molecule. Using molecular dynamics simulation, we elucidated the molecular details of Ritonavir-ALKBH2 interaction. The present work highlights that Ritonavir might be used to target the ALKBH2-mediated DNA alkylation repair.
\end{abstract}




\section{INTRODUCTION}

DNA alkylating agents are found abundantly in industrial chemicals, environmental contaminants, and used as cancer chemotherapeutic drugs. DNA alkylating drug Temozolomide (TMZ) is an approved drug for glioblastoma multiforme (GBM) and astrocytoma which are the most common primary brain tumour in adults for which complete cure remains elusive. TMZ is also used in malignant melanoma. TMZ generates highly reactive methyl-diazonium cation that mainly targets Oxygen and Nitrogen atoms of the DNA bases (Hecht \& Hoffmann 1988; Margison, Santibanez Koref \& Povey 2002). Although effective in killing cancer cells, most chemotherapeutic alkylating agents have two important limitations: dose-limiting toxicity and resistance development. Thus, there is a critical need to improve the sensitivity of DNA alkylating drugs. The reaction of duplex DNA with TMZ results in N7-methylguanine (7meG), N3-methyl Adenine (3meA) and O6-methylguanine (6meG). TMZ exposure in single-stranded DNA (ssDNA) results in two additional adducts; namely, N1-methyladenine (1meA) and N3-methylcytosine (3meC) (Sedgwick 2004). Several DNA repair mechanisms can facilitate TMZ resistance and cancer cell survival by removing TMZ-induced DNA adducts. For instance, $6 \mathrm{meG}$ adducts are substrates for the ubiquitous DNA repair protein O6-methylguanine-DNA methyltransferase (MGMT), whereas the 3-meA adducts are repaired by the base excision repair (BER) (Johannessen, Bjerkvig \& Tysnes 2008). While $7 \mathrm{meG}$ is well-tolerated, $3 \mathrm{meC}$ and $1 \mathrm{meA}$ adducts are repaired directly by $\mathrm{Fe}^{2+}$ and 2-oxoglutarate-dependent dioxygenases. This class of enzyme was first discovered in E. coli and known as Alkylation repair protein-B (AlkB) (Wyatt \& Pittman 2006). AlkB, which is highly conserved from $E$. coli to humans, catalyses direct reversal of $1 \mathrm{meA}$ and $3 \mathrm{meC}$ by an oxidative demethylation reaction (Falnes, Johansen \& Seeberg 2002; Trewick, Henshaw, Hausinger, Lindahl \& Sedgwick 2002). Mammalian cells have at least eight AlkB family members (ALKBH1-8) and only ALKBH2 and ALKBH3 have been shown to possess DNA repair activity similar to the E. coli AlkB (Lee et al. 2005). In human cells, ALKBH2 demethylase associates with proliferating cell nuclear antigen (PCNA) (Ringvoll et al. 2006; Nay, Lee, Bates \& O'Connor 2012). With its strong preference for double-stranded DNA, ALKBH2 uses facilitated one-dimensional diffusion and lateral movement along the helix to scour the genome for alky adducts (Aas et al. 2003; Chen, Liu, Sun \& Yang 2010). The other AlkB homologue, ALKBH3, prefers SsDNA substrates and requires DNA unwinding activity of a DNA helicase known as activating signal cointegrator complex-3 (ASCC3) to demethylate the genomic DNA (Aas et al. 2003; Dango et al. 2011; Fu, Samson, Hubscher \& van Loon 2015). Preclinical in vitro studies suggest that MGMT is the major cause of the resistance of tumour cells to the cytocidal effects of TMZ (Pegg 1990). However, the link between the expression of MGMT and the sensitivity of cells to TMZ is not as clear in clinical studies. Tumours that are currently treated with TMZ and similar alkylating 
agents, such as glioblastoma or melanoma, have the lowest MGMT expression (Middleton \& Margison 2003). The anticancer efficacy of DNA alkylating drug TMZ on GBM cell lines was shown to be inhibited by the expression of DNA repair enzyme ALKBH2 (Johannessen et al. 2013). Overexpression of ALKBH2 in GBM cell lines results in cellular resistance to TMZ and MMS and silencing of ALKBH2 increases cellular sensitivity to both TMZ and MMS (Johannessen et al. 2013), suggesting that a high level of ALKBH2 expression could be an important mediator of chemo-resistance in human GBM. ALKBH2 gene is also known to overexpress in NSCLC and bladder cancer (Wu et al. 2011; Fujii, Shimada, Anai, Fujimoto \& Konishi 2013). It was reported that silencing of the ALKBH2 gene sensitizes NSCLC cells to cisplatin by growth inhibition and the induction of apoptosis (Wu et al. 2011). Knockdown of ALKBH2 resulted in cell cycle arrest in bladder cancer cells (Fujii et al. 2013). Therefore, DNA alkylation repair by ALKBH2 can enable various tumour cells to survive DNA damage that is induced by DNA damaging chemotherapeutic treatments; therefore, inhibitors of ALKBH2 might prove efficacious when used in combination with DNA-damaging chemotherapeutic drugs. Therefore, new therapeutic approaches targeting the ALKBH2 mediated DNA repair mechanisms to inhibit cancer cell growth and survival requires urgent attention.

Multiple reports suggest that Ritonavir has anti-tumour activity against various types of cancers. The human immunodeficiency virus (HIV) infection is characterized by an increased risk of several solid tumours due to its inherent nature of weakening the immune system. However, recent observations suggest a lower incidence of malignancies in patients infected with HIV during treatment with Highly Active Anti-Retroviral Therapy (HAART) utilizing protease inhibitors. Ritonavir, an FDA approved HIV protease inhibitor, has nonHIV-related anti-tumorigenic activity that has been attributed to multiple mechanisms, including (a) metabolic stress due to inhibition of glucose uptake (Murata, Hruz \& Mueckler 2000; Ikezoe et al. 2004; Kumar et al. 2009; McBrayer et al. 2012; Adekola et al. 2015; Mishra et al. 2015; Bajpai et al. 2016); (b) impaired cell invasion due to inhibition matrix metalloprotease activity (Laurent et al. 2004; Ahluwalia et al. 2011; Barillari et al. 2012); (c) ER stress due to modulation of the proteasome function (Maggiorella et al. 2005; Kraus et al. 2008; Sato et al. 2010; Kraus et al. 2014; Liu et al. 2015; Sato, Asano, Okubo, Isono \& Asano 2017; Isono, Sato, Asano, Okubo \& Asano 2018) and NFאB signalling (Dewan et al. 2006). Clinical trials with Ritonavir revealed that it has promising anti-tumour activity results against advanced pancreatic cancer (Brunner et al. 2008) and solid tumour when combined with docetaxel (de Weger et al. 2017). Another clinical trial showed the direct topical application of Ritonavir to the cervix prevented HPV related cervical dysplasia (Hampson et al. 2016). Currently, a trial (NCT02770378) is ongoing studying the combined effect of ritonavir with aprepitant, artesunate, auranofin, captopril, celecoxib, disulfiram, itraconazole, 
sertraline and continuous low dose temozolomide in glioblastoma. Phase II trial on dose escalation studies on ritonavir as an anti-tumour agent established that it is tolerated at high doses and higher doses could yield greater clinical responses (de Weger et al. 2017). Drug repurposing is an efficient approach for discovering new uses from already existing drugs. There are various examples of repurposed drugs that were discovered by computational approach and are now being used in other diseases. For example, Raltegravir, originally an HIV-1 integrase inhibitor is now used as adjuvant therapy in cancer and Valsartan, an angiotensin receptor blocker is now being used for Alzheimer's disease (Oprea et al. 2011; Lee \& Kim 2016). To explore if FDA approved HIV protease inhibitor drug Ritonavir could be repurposed as an inhibitor of the DNA repair enzyme ALKBH2, HIV protease inhibitor drugs were screened and Ritonavir was found to be a likely inhibitor against ALKBH2.

\section{RESULTS AND DISCUSSION}

\section{In silico docking studies of FDA-approved HIV protease inhibitors}

To explore if FDA approved HIV protease inhibitor drug Ritonavir could be repurposed as an inhibitor of DNA repair enzyme ALKBH2, we have screened Ritonavir along with 12 HIV protease inhibitor drugs using molecular docking using Glide/XP module of Schrodinger. For comparison we have included ALKBH3. Out of the screened drugs, Ritonavir showed the lowest binding energy for ALKBH2 (Table 1) and we proceeded with this complex for further analysis. From the ten likely conformations for each Ritonavir complex, the lowest energy conformation of ALKBH2-Ritonavir and ALKBH3-Ritonavir complexes were selected (Figure 1A).

\section{Molecular docking reveals Ritonavir occluding the catalytic site of ALKBH2}

To study the Ritonavir binding in more detail, potential binding sites on ALKBH2 and ALKBH3 was identified using the SiteMap module of Maestro (Halgren et al. 2004). The Sitecore is an empirical function representing the weighted sum of hydrogen bonding, exposure, contact, and hydrophilic or hydrophobic parameters of the protein. In general, a SiteScore of $>1.0$ is associated with a site that is considered druggable and a score $<1.0$ indicates that the region is difficult for binding, and is considered as non-druggable. Sitescores of AlkBH2 and ALKBH3 were 1.028 and 1.061 respectively. This indicates that Ritonavir could bind to both ALKBH2 and ALKBH3. GLIDE molecular docking output which is known as GlideScore is a scoring function that calculates the ligand-protein interaction energies, hydrogen bonding, hydrophobic interactions, and $\pi-\pi$ stacking interactions. It was observed that GlideScore of ALKBH2-Ritonavir and ALKBH3-Ritonavir complexes were $6.278 \mathrm{kcal} / \mathrm{mol}$ and $-6.161 \mathrm{kcal} / \mathrm{mol}$ respectively. Since the GlideScore of both ALKBH2 and ALKBH3 were almost similar, this indicates that both ALKBH2 and ALKBH3 could bind Ritonavir with equal affinity. Ligand- 
Protein interaction analysis revealed that Ritonavir could form a hydrogen bond with Ser125 and Glu125 from ALKBH2. Interestingly, the previously reported crystal structure of ALKBH2 in complex with $\mathrm{m}^{1} \mathrm{~A}$-containing dsDNA shows that Ser125 is one of the key residues that form a hydrogen bond with the substrate (Halgren 2009). This indicates that the binding of Ritonavir in the active site pocket of ALKBH2 might interfere with DNA binding and might act as a competitive inhibitor. In addition, docking analysis of ALKBH2-Ritonavir shows $\pi-\pi$ stacking interaction with His106 and hydrophobic interactions with several residues namely; Val99, Phe102, Val108, Phe124, Ile168 and Val240. In the case of the ALKBH3-Ritonavir complex, it was observed that Ritonavir forms a hydrogen bond with Asp124 and Ile121 residues. The highly conserved $\mathrm{H}-\mathrm{X}-\mathrm{D}-\mathrm{Xn}$-H motif of ALKBH3 is comprised of His191, Asp193 and His257 residues (Sundheim et al. 2006). Interestingly, it was noted that Ritonavir forms $\pi-\pi$ stacking interaction with catalytically active site residue His 191 . Also, Ritonavir was found to be involved in hydrophobic interaction with Trp135, Met148, Val188 and Pro196 residues of ALKBH3.

\section{Molecular dynamics simulation reveals stable interaction of Ritonavir with ALKBH2}

The stability of the Ritonavir-ALKBH2 structure obtained from the docking calculations was assessed by evaluating the stability of the complex via MD simulations. For comparison, the Ritonavir-ALKBH3 complex was also monitored. Each complex was subjected to $100 \mathrm{~ns}$ MD simulation. MD simulation was performed using the Desmond package to validate the plausibility of ALKBH2-Ritonavir and ALKBH3-Ritonavir docked complex obtained from GLIDE docking. RMSD analysis shows that the ALKBH2-Ritonavir complex maintained a stable conformation with very less fluctuations (Figure 2A and $\mathbf{C}$ ). The average RMSD of Ritonavir concerning ALKBH2 was found to be 5.9A. The mean Ca RMSD of ALKBH2 over 100 ns was found to be $2 \AA$ indicating that the binding of Ritonavir did not result in any large conformational changes in ALKBH2 structure during the simulation. Whereas, in the case of ALKBH3-Ritonavir complex there were some fluctuations until 60 ns. But, after 60 ns there was a significantly larger fluctuation as evident from an increase in RMSDs from 8.5 to 19.7 $\AA$ over the 100 ns run time. Also, the mean RMSD of Ritonavir concerning ALKBH3 was found to be $12.99 \AA$ which indicates that Ritonavir has diffused away from its initial binding site.

Protein-Ligand interaction analysis shows that in the case of the ALKBH2-Ritonavir complex, Ritonavir forms a hydrogen bond with Ser125 throughout the trajectory (Figure 2B). Interestingly, Ritonavir also forms a water-mediated hydrogen bond with Arg110, which is yet another key residue in ALKBH2 important for dsDNA binding. In addition to this, hydrophobic contacts with Phe124 and lle169 was also observed over the trajectory. Whereas in the case of ALKBH3-Ritonavir complex, interaction with catalytically active site residue His191 was lost over the trajectory (Figure 2D). Also, water-mediated contact was 
observed with Pro140 and Ser144 residues which were present away from the active site pocket. From the MM-GBSA analysis, it was found that at $100 \mathrm{~ns}$ ALKBH2-Ritonavir complex had binding free energy of $-74.0 \mathrm{kcal} / \mathrm{mol}$, whereas ALKBH3-Ritonavir exhibited binding free energy of $-40.50 \mathrm{kcal} / \mathrm{mol}$. This indicates that the Ritonavir complex binds energetically favourable at the active site of ALKBH2 when compared to ALKBH3. The conformational and structural changes along the simulation trajectories that ultimately allow ALKBH2-Ritonavir and ALKBH3-Ritonavir binding is shown in supplementary information (Movie S1 and S2). From Movie S1, it is also clear that the ligand Ritonavir remained in the initial binding pocket of ALKBH2 throughout the simulation time. However, in the RitonavirALKBH3 complex, the ligand moved away from the initial binding site and became exposed to the solvent (Movie S2). The analysis of contacts reveals that Ritonavir moved from the initial binding site to different cavities of the protein and sometimes outside the protein, and therefore, formed contacts with very many residues of the protein.

In summary, these MD simulations represent a case of stronger binding of Ritonavir to $A L K B H 2$ and weaker binding for ALKBH3. The conformation of the ALKBH2 bound Ritonavir system is comparatively more stabilized during the MD simulation.

\section{MATERIALS AND METHODS}

\section{Molecular docking using GLIDE}

The three-dimensional structure of ALKBH2 (3S57) and ALKBH3 (2IUW) was retrieved from RCSB Protein Data Bank. The protein structures were refined using Protein-preparation wizard in Maestro, Schrödinger, LLC, 2018 suite to resolve the stereochemical errors [1]. This involves assignment of proper bond order, elimination of steric clashes, addition of hydrogen atoms, fixing the disulfide bonds, missing residues and loop regions. Later, hydrogen bond assignment was done using ProtAssign. In addition, the protein structure was also optimized and the protonation states of charged residues were determined using PROPKA Schrodinger's Maestro suite. Finally, to obtain optimal geometry of the proteins, energy minimization was performed using OPLS-2005 (Optimized Potentials for Liquid Simulations) force field (Harder et al. 2016). The three-dimensional structure of Ritonavir was subjected to the Ligprep for the ligand preparation. The prepared structure of Ritonavir was then optimized and energy minimized using OPLS 2005 force field to acquire an energetically stable geometry of the compound. SiteMap module of Maestro was used to delineate the potential binding sites in ALKBH2 and ALKBH3 protein. A standard grid was generated over the whole target protein by SiteMap. Ritonavir was docked to ALKBH2 and ALKBH3 using Glide/XP module of Schrodinger (Halgren et al. 2004).

\section{Molecular Dynamics (MD) simulations}


Molecular dynamics simulations of ALKBH2-Ritonavir and ALKBH3-Ritonavir docked complex was performed using Desmond package. Using System Builder panel, the protein was solvated with TIP3P (transferable intermolecular potential with 3 points) water model in an orthorhombic box of dimension $20 \AA \times 20 \AA \times 20 \AA$. The system was neutralized by adding $11 \mathrm{Cl}^{-}$and $2 \mathrm{Cl}^{-}$counter ions for $\mathrm{ALKBH} 2$ and $\mathrm{ALKBH} 3$ respectively. Also, the ionic strength was maintained by adding a physiological concentration of $0.15 \mathrm{M} \mathrm{NaCl}$ to the simulation box. The total number of atoms in the simulation boxes for ALKBH2 and ALKBH3 are 275901 and 271750 respectively. In addition, the system was equilibrated with the NPT ensemble, by setting constant temperature and pressure at $300 \mathrm{~K}$ and 1.01325 bar, respectively. Finally, the well-equilibrated system with all the atoms was subjected to a production run of $100 \mathrm{~ns}$. The binding energy of protein-ligand complexes was estimated using the prime molecular mechanics-generalized born surface area (MM-GBSA) module in the Schrodinger Suite 2018. The quality of MD simulations was analysed by the Simulation Event Analysis tool in Maestro.

\section{Supplementary Information:}

The conformational and structural changes along the simulation trajectories repreenting ALKBH2-Ritonavir and ALKBH3-Ritonavir binding is shown in Supplementary information Movie S1 and S2.

\section{Acknowledgement}

This work was supported by Science and Engineering Research Board (SERB), grant EMR/2016/005135/BBM.

\section{Conflict of Interest}

The authors declare that they have no conflicts of interest with the contents of this article.

\section{Reference}

Aas, P. A., Otterlei, M., Falnes, P. O., Vagbo, C. B., Skorpen, F., Akbari, M., Sundheim, O., Bjoras, M., Slupphaug, G., Seeberg, E. \& Krokan, H. E. (2003). Human and bacterial oxidative demethylases repair alkylation damage in both RNA and DNA. Nature 421: 859-863.

Adekola, K. U., Dalva Aydemir, S., Ma, S., Zhou, Z., Rosen, S. T. \& Shanmugam, M. (2015). Investigating and targeting chronic lymphocytic leukemia metabolism with the human immunodeficiency virus protease inhibitor ritonavir and metformin. Leuk Lymphoma 56: 450-459. 
Ahluwalia, M. S., Patton, C., Stevens, G., Tekautz, T., Angelov, L., Vogelbaum, M. A., Weil, R. J., Chao, S., Elson, P., Suh, J. H., Barnett, G. H. \& Peereboom, D. M. (2011). Phase II trial of ritonavir/lopinavir in patients with progressive or recurrent high-grade gliomas. J Neurooncol 102: 317-321.

Bajpai, R., Matulis, S. M., Wei, C., Nooka, A. K., Von Hollen, H. E., Lonial, S., Boise, L. H. \& Shanmugam, M. (2016). Targeting glutamine metabolism in multiple myeloma enhances BIM binding to BCL-2 eliciting synthetic lethality to venetoclax. Oncogene 35: 3955-3964.

Barillari, G., Iovane, A., Bacigalupo, I., Palladino, C., Bellino, S., Leone, P., Monini, P. \& Ensoli, B. (2012). Ritonavir or saquinavir impairs the invasion of cervical intraepithelial neoplasia cells via a reduction of MMP expression and activity. AIDS 26: 909-919.

Brunner, T. B., Geiger, M., Grabenbauer, G. G., Lang-Welzenbach, M., Mantoni, T. S., Cavallaro, A., Sauer, R., Hohenberger, W. \& McKenna, W. G. (2008). Phase I trial of the human immunodeficiency virus protease inhibitor nelfinavir and chemoradiation for locally advanced pancreatic cancer. J Clin Oncol 26: 2699-2706.

Chen, B., Liu, H., Sun, X. \& Yang, C. G. (2010). Mechanistic insight into the recognition of single-stranded and double-stranded DNA substrates by $\mathrm{ABH} 2$ and $\mathrm{ABH}$. $\mathrm{Mol}$ Biosyst 6: 2143-2149.

Dango, S., Mosammaparast, N., Sowa, M. E., Xiong, L. J., Wu, F., Park, K., Rubin, M., Gygi, S., Harper, J. W. \& Shi, Y. (2011). DNA unwinding by ASCC3 helicase is coupled to ALKBH3-dependent DNA alkylation repair and cancer cell proliferation. Mol Cell 44: 373-384.

de Weger, V. A., Stuurman, F. E., Hendrikx, J., Moes, J. J., Sawicki, E., Huitema, A. D. R., Nuijen, B., Thijssen, B., Rosing, H., Keessen, M., Mergui-Roelvink, M., Beijnen, J. H., Schellens, J. H. M. \& Marchetti, S. (2017). A dose-escalation study of bi-daily once weekly oral docetaxel either as ModraDoc001 or ModraDoc006 combined with ritonavir. Eur J Cancer 86: 217-225.

Dewan, M. Z., Uchihara, J. N., Terashima, K., Honda, M., Sata, T., Ito, M., Fujii, N., Uozumi, K., Tsukasaki, K., Tomonaga, M., Kubuki, Y., Okayama, A., Toi, M., Mori, N. \& Yamamoto, N. (2006). Efficient intervention of growth and infiltration of primary adult T-cell leukemia cells by an HIV protease inhibitor, ritonavir. Blood 107: 716724.

Falnes, P. O., Johansen, R. F. \& Seeberg, E. (2002). AlkB-mediated oxidative demethylation reverses DNA damage in Escherichia coli. Nature 419: 178-182.

Fu, D., Samson, L. D., Hubscher, U. \& van Loon, B. (2015). The interaction between ALKBH2 DNA repair enzyme and PCNA is direct, mediated by the hydrophobic pocket of PCNA and perturbed in naturally-occurring ALKBH2 variants. DNA Repair (Amst) 35: 13-18.

Fujii, T., Shimada, K., Anai, S., Fujimoto, K. \& Konishi, N. (2013). ALKBH2, a novel AlkB homologue, contributes to human bladder cancer progression by regulating MUC1 expression. Cancer Sci 104: 321-327.

Halgren, T. A. (2009). Identifying and characterizing binding sites and assessing druggability. J Chem Inf Model 49: 377-389.

Halgren, T. A., Murphy, R. B., Friesner, R. A., Beard, H. S., Frye, L. L., Pollard, W. T. \& Banks, J. L. (2004). Glide: a new approach for rapid, accurate docking and scoring. 2. Enrichment factors in database screening. J Med Chem 47: 1750-1759. 
Hampson, L., Maranga, I. O., Masinde, M. S., Oliver, A. W., Batman, G., He, X., Desai, M., Okemwa, P. M., Stringfellow, H., Martin-Hirsch, P., Mwaniki, A. M., Gichangi, P. \& Hampson, I. N. (2016). A Single-Arm, Proof-Of-Concept Trial of Lopimune (Lopinavir/Ritonavir) as a Treatment for HPV-Related Pre-Invasive Cervical Disease. PLoS One 11: e0147917.

Harder, E., Damm, W., Maple, J., Wu, C., Reboul, M., Xiang, J. Y., Wang, L., Lupyan, D., Dahlgren, M. K., Knight, J. L., Kaus, J. W., Cerutti, D. S., Krilov, G., Jorgensen, W. L., Abel, R. \& Friesner, R. A. (2016). OPLS3: A Force Field Providing Broad Coverage of Drug-like Small Molecules and Proteins. J Chem Theory Comput 12: 281-296.

Hecht, S. S. \& Hoffmann, D. (1988). Tobacco-specific nitrosamines, an important group of carcinogens in tobacco and tobacco smoke. Carcinogenesis 9: 875-884.

Ikezoe, T., Saito, T., Bandobashi, K., Yang, Y., Koeffler, H. P. \& Taguchi, H. (2004). HIV-1 protease inhibitor induces growth arrest and apoptosis of human multiple myeloma cells via inactivation of signal transducer and activator of transcription 3 and extracellular signal-regulated kinase 1/2. Mol Cancer Ther 3: 473-479.

Isono, M., Sato, A., Asano, T., Okubo, K. \& Asano, T. (2018). Delanzomib Interacts with Ritonavir Synergistically to Cause Endoplasmic Reticulum Stress in Renal Cancer Cells. Anticancer Res 38: 3493-3500.

Johannessen, T. C., Bjerkvig, R. \& Tysnes, B. B. (2008). DNA repair and cancer stem-like cells-potential partners in glioma drug resistance? Cancer Treat Rev 34: 558-567.

Johannessen, T. C., Prestegarden, L., Grudic, A., Hegi, M. E., Tysnes, B. B. \& Bjerkvig, R. (2013). The DNA repair protein ALKBH2 mediates temozolomide resistance in human glioblastoma cells. Neuro Oncol 15: 269-278.

Kraus, M., Malenke, E., Gogel, J., Muller, H., Ruckrich, T., Overkleeft, H., Ovaa, H., Koscielniak, E., Hartmann, J. T. \& Driessen, C. (2008). Ritonavir induces endoplasmic reticulum stress and sensitizes sarcoma cells toward bortezomib-induced apoptosis. Mol Cancer Ther 7: 1940-1948.

Kraus, M., Muller-Ide, H., Ruckrich, T., Bader, J., Overkleeft, H. \& Driessen, C. (2014). Ritonavir, nelfinavir, saquinavir and lopinavir induce proteotoxic stress in acute myeloid leukemia cells and sensitize them for proteasome inhibitor treatment at low micromolar drug concentrations. Leuk Res 38: 383-392.

Kumar, S., Bryant, C. S., Chamala, S., Qazi, A., Seward, S., Pal, J., Steffes, C. P., Weaver, D. W., Morris, R., Malone, J. M., Shammas, M. A., Prasad, M. \& Batchu, R. B. (2009). Ritonavir blocks AKT signaling, activates apoptosis and inhibits migration and invasion in ovarian cancer cells. Mol Cancer 8: 26.

Laurent, N., de Bouard, S., Guillamo, J. S., Christov, C., Zini, R., Jouault, H., Andre, P., Lotteau, V. \& Peschanski, M. (2004). Effects of the proteasome inhibitor ritonavir on glioma growth in vitro and in vivo. Mol Cancer Ther 3: 129-136.

Lee, D. H., Jin, S. G., Cai, S., Chen, Y., Pfeifer, G. P. \& O'Connor, T. R. (2005). Repair of methylation damage in DNA and RNA by mammalian AlkB homologues. $J$ Biol Chem 280: 39448-39459.

Lee, H. M. \& Kim, Y. (2016). Drug Repurposing Is a New Opportunity for Developing Drugs against Neuropsychiatric Disorders. Schizophr Res Treatment 2016: 6378137.

Liu, R., Zhang, L., Yang, J., Zhang, X., Mikkelsen, R., Song, S. \& Zhou, H. (2015). HIV Protease Inhibitors Sensitize Human Head and Neck Squamous Carcinoma Cells to Radiation by Activating Endoplasmic Reticulum Stress. PLoS One 10: e0125928. 
Maggiorella, L., Wen, B., Frascogna, V., Opolon, P., Bourhis, J. \& Deutsch, E. (2005). Combined radiation sensitizing and anti-angiogenic effects of ionizing radiation and the protease inhibitor ritonavir in a head and neck carcinoma model. Anticancer Res 25: 4357-4362.

Margison, G. P., Santibanez Koref, M. F. \& Povey, A. C. (2002). Mechanisms of carcinogenicity/chemotherapy by 06-methylguanine. Mutagenesis 17: 483-487.

McBrayer, S. K., Cheng, J. C., Singhal, S., Krett, N. L., Rosen, S. T. \& Shanmugam, M. (2012). Multiple myeloma exhibits novel dependence on GLUT4, GLUT8, and GLUT11: implications for glucose transporter-directed therapy. Blood 119: 4686-4697.

Middleton, M. R. \& Margison, G. P. (2003). Improvement of chemotherapy efficacy by inactivation of a DNA-repair pathway. Lancet Oncol 4: 37-44.

Mishra, R. K., Wei, C., Hresko, R. C., Bajpai, R., Heitmeier, M., Matulis, S. M., Nooka, A. K., Rosen, S. T., Hruz, P. W., Schiltz, G. E. \& Shanmugam, M. (2015). In Silico Modelingbased Identification of Glucose Transporter 4 (GLUT4)-selective Inhibitors for Cancer Therapy. J Biol Chem 290: 14441-14453.

Murata, H., Hruz, P. W. \& Mueckler, M. (2000). The mechanism of insulin resistance caused by HIV protease inhibitor therapy. J Biol Chem 275: 20251-20254.

Nay, S. L., Lee, D. H., Bates, S. E. \& O'Connor, T. R. (2012). Alkbh2 protects against lethality and mutation in primary mouse embryonic fibroblasts. DNA Repair (Amst) 11: 502510.

Oprea, T. I., Bauman, J. E., Bologa, C. G., Buranda, T., Chigaev, A., Edwards, B. S., Jarvik, J. W., Gresham, H. D., Haynes, M. K., Hjelle, B., Hromas, R., Hudson, L., Mackenzie, D. A., Muller, C. Y., Reed, J. C., Simons, P. C., Smagley, Y., Strouse, J., Surviladze, Z., Thompson, T., Ursu, O., Waller, A., Wandinger-Ness, A., Winter, S. S., Wu, Y., Young, S. M., Larson, R. S., Willman, C. \& Sklar, L. A. (2011). Drug Repurposing from an Academic Perspective. Drug Discov Today Ther Strateg 8: 61-69.

Pegg, A. E. (1990). Mammalian O6-alkylguanine-DNA alkyltransferase: regulation and importance in response to alkylating carcinogenic and therapeutic agents. Cancer Res 50: 6119-6129.

Ringvoll, J., Nordstrand, L. M., Vagbo, C. B., Talstad, V., Reite, K., Aas, P. A., Lauritzen, K. H., Liabakk, N. B., Bjork, A., Doughty, R. W., Falnes, P. O., Krokan, H. E. \& Klungland, A. (2006). Repair deficient mice reveal $\mathrm{mABH} 2$ as the primary oxidative demethylase for repairing 1meA and 3meC lesions in DNA. EMBO J 25: 2189-2198.

Sato, A., Asano, T., Horiguchi, A., Ito, K., Sumitomo, M. \& Asano, T. (2010). Combination of suberoylanilide hydroxamic acid and ritonavir is effective against renal cancer cells. Urology 76: 764 e767-713.

Sato, A., Asano, T., Okubo, K., Isono, M. \& Asano, T. (2017). Ritonavir and ixazomib kill bladder cancer cells by causing ubiquitinated protein accumulation. Cancer Sci 108: 1194-1202.

Sedgwick, B. (2004). Repairing DNA-methylation damage. Nat Rev Mol Cell Biol 5: 148-157.

Sundheim, O., Vagbo, C. B., Bjoras, M., Sousa, M. M., Talstad, V., Aas, P. A., Drablos, F., Krokan, H. E., Tainer, J. A. \& Slupphaug, G. (2006). Human ABH3 structure and key residues for oxidative demethylation to reverse DNA/RNA damage. EMBO J 25: 3389-3397.

Trewick, S. C., Henshaw, T. F., Hausinger, R. P., Lindahl, T. \& Sedgwick, B. (2002). Oxidative demethylation by Escherichia coli AlkB directly reverts DNA base damage. Nature 419: 174-178. 
Wu, S. S., Xu, W., Liu, S., Chen, B., Wang, X. L., Wang, Y., Liu, S. F. \& Wu, J. Q. (2011). Downregulation of ALKBH2 increases cisplatin sensitivity in $\mathrm{H} 1299$ lung cancer cells. Acta Pharmacol Sin 32: 393-398.

Wyatt, M. D. \& Pittman, D. L. (2006). Methylating agents and DNA repair responses: Methylated bases and sources of strand breaks. Chem Res Toxicol 19: 1580-1594.

A
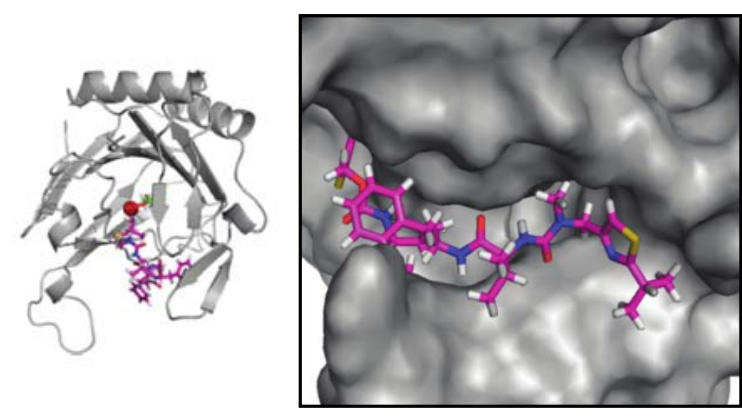

C

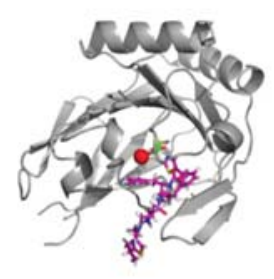

B

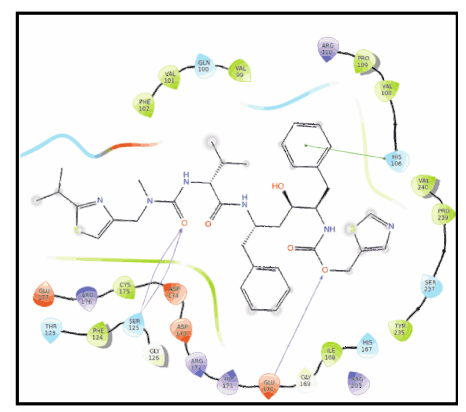

D

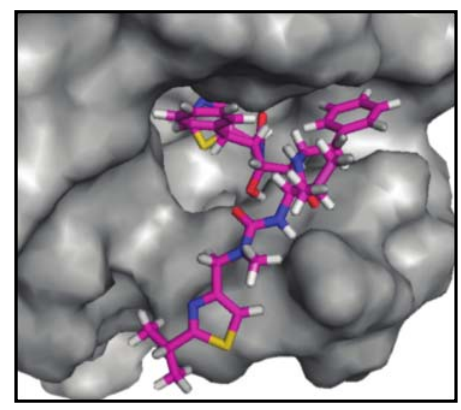

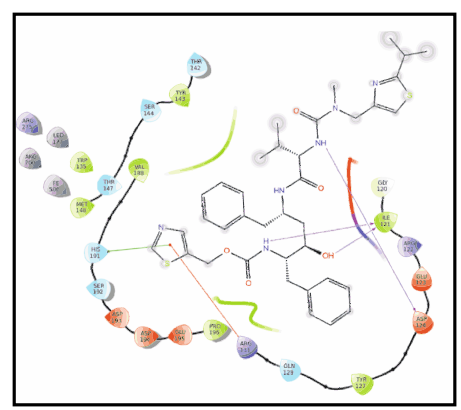

Figure 1. Molecular docking study of Ritonavir. (A) A representative complex of Ritonavir with $\mathrm{ALKBH} 2$ (B) 2D ligand interaction diagram show key interactions at the binding site. (C) A representative complex of Ritonavir with ALKBH3 (D) 2D ligand interaction diagram show key interactions at the binding site. 
A

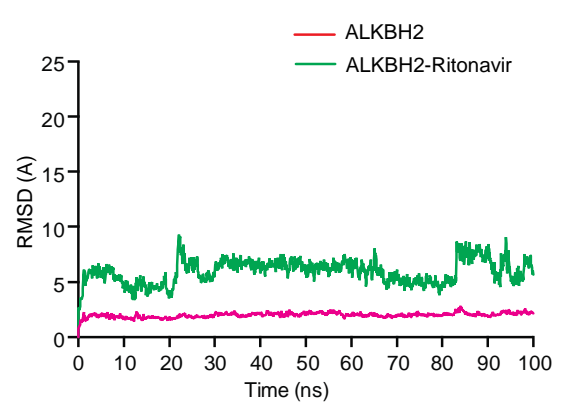

C

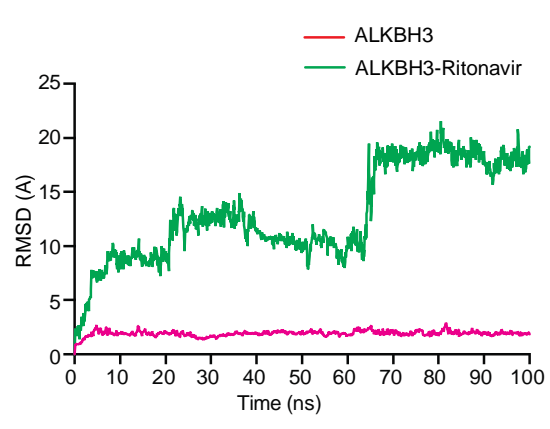

B

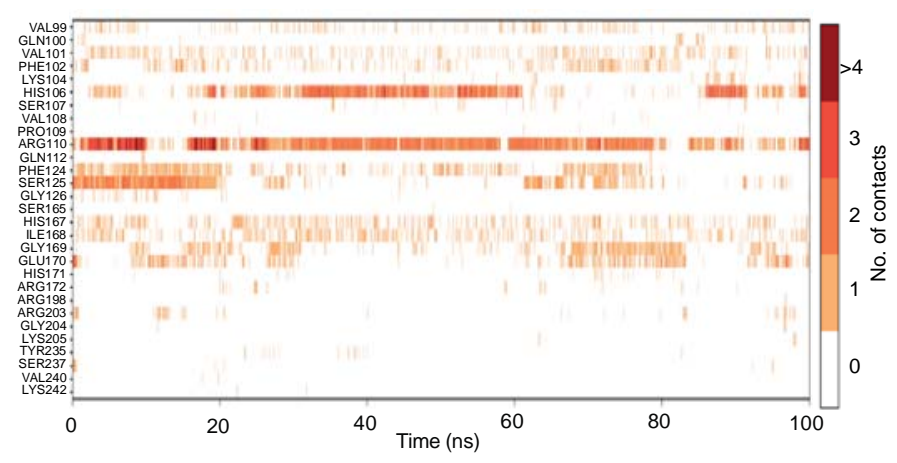

D

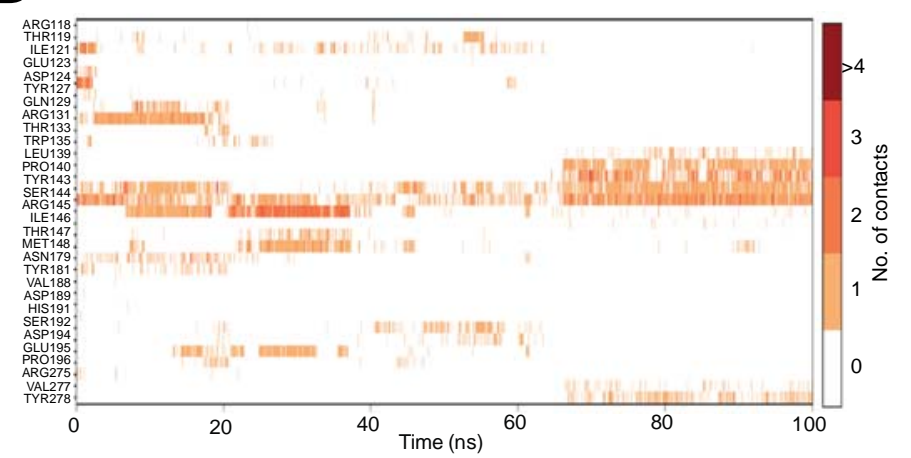

Figure 2. Molecular dynamics simulation of interaction of Ritonavir with ALKBH2 and ALKBH3. (A) The Root means squared deviations (RMSD) of Ca atoms of comparative 'apo' and 'holo' forms of ALKBH2 in the course of $0.1 \mu \mathrm{s}$ MD simulations. (B) Non-covalent bond interactions between Ritonavir with the binding pocket amino acid residues of ALKBH2. (C) RMSD of Ca atoms of comparative 'apo' and 'holo' forms of ALKBH3 (D) Non-covalent bond interactions between Ritonavir with the binding pocket amino acid residues of ALKBH3. 
bioRxiv preprint doi: https://doi.org/10.1101/2021.09.26.461894; this version posted September 27, 2021. The copyright holder for this preprint (which was not certified by peer review) is the author/funder, who has granted bioRxiv a license to display the preprint in perpetuity. It is made available under aCC-BY-ND 4.0 International license.

TABLE 1. Docking score of FDA approved HIV protease inhibitor drugs

\begin{tabular}{|c|c|c|c|c|}
\hline \multirow[t]{2}{*}{ Drug } & \multicolumn{2}{|c|}{ ALKBH3 } & \multicolumn{2}{|c|}{ ALKBH2 } \\
\hline & $\begin{array}{c}\text { Docking } \\
\text { Full Fitness } \\
\text { (kcal/mol) }\end{array}$ & $\begin{array}{c}\text { Binding energy } \\
\text { Estimated } \Delta \mathrm{G} \\
(\mathrm{kcal} / \mathrm{mol})\end{array}$ & $\begin{array}{c}\text { Docking } \\
\text { Full Fitness } \\
\text { (kcal/mol) }\end{array}$ & $\begin{array}{c}\text { Binding } \\
\text { energy } \\
\text { Estimated } \Delta \mathrm{G} \\
(\mathrm{kcal} / \mathrm{mol})\end{array}$ \\
\hline Atazanavir & -1173.72 & -9.67 & -1074.61 & -8.94 \\
\hline Darunavir & -1257.36 & -8.47 & -1163.61 & -8.55 \\
\hline Fosamprenavir & -1384.37 & -6.73 & -1299.58 & -9.70 \\
\hline Ritonavir & -1294.96 & -9.69 & -1203.27 & -11.37 \\
\hline Saquinavir & -1145.46 & -9.40 & -1054.98 & -9.21 \\
\hline Tipranavir & -1208.21 & -10.58 & -1113.12 & -9.46 \\
\hline Amprenavir & -1252.19 & -8.12 & -1161.87 & -8.55 \\
\hline lopinavir & -1227.01 & -9.18 & -1116.61 & -8.24 \\
\hline Indinavir & -1135.07 & -9.92 & -1030.23 & -9.02 \\
\hline Nelfinavir & -1179.46 & -8.96 & -1071.55 & -7.59 \\
\hline
\end{tabular}

